\title{
Lymphoid infiltrates in B cell non Hodgkin's lymphoma: Comparing nuclear characteristics between lymph node and bone marrow; and evaluating diagnostic features of bone marrow infiltrates in paraffin embedded tissues
}

\author{
Mark H. Deverell ${ }^{1}$, Elizabeth Best ${ }^{2}$ and Jonathan R. Salisbury ${ }^{2, *}$ \\ ${ }^{1}$ The Health Centre, Marylebone Road, March, Cambridge, UK \\ ${ }^{2}$ Department of Histopathology, King's College School of Medicine, London SE5 9PJ, UK
}

Received 23 January 1996

Revised 21 January 1997

\begin{abstract}
Distinguishing non Hodgkin's lymphoma from benign lymphoid aggregates in bone marrow is well recognised to be difficult. Our objective was to evaluate nuclear morphology, and to perform morphometry on benign and neoplastic lymphoid infiltrates, to establish if objective criteria were of value in the diagnosis of neoplasia. By comparing neoplastic infiltrates in bone marrow with infiltrates in lymph nodes, the validity of grading non Hodgkin's lymphoma on the basis of bone marrow histology alone was assessed. 82 cases of B cell non Hodgkin's lymphoma (44 low grade and 38 high grade), known to have both lymph node and bone marrow involvement at the time of presentation, were compared with bone marrow trephines containing reactive lymphoid infiltrates.

The results suggest that in paraffin embedded tissue from bone marrow trephines, nuclear morphology, nuclear area and the nuclear contour index cannot, in most cases, be used to distinguish reactive from neoplastic lymphoid infiltrates although mean nuclear area supports a diagnosis of a neoplastic infiltrate if it can be shown that the nuclei are larger than would be expected in reactive infiltrates. Such differences are subtle and often not appreciable without the use of quantitative techniques. The frequent occurrence of discordant infiltrates in high grade lymphomas means grading of lymphoma on the basis of bone marrow appearances is often unrealistic.
\end{abstract}

Keywords: Non Hodgkin's lymphoma, bone marrow, karyometry, computer assisted image processing

\footnotetext{
*Address for correspondence: Dr. J.R. Salisbury, Department of Histopathology, King's College School of Medicine, Bessemer Road, London SE5 9PJ, UK.
} 


\section{Introduction}

The distinction of non Hodgkin's lymphoma from benign lymphoid aggregates in bone marrow trephines is difficult and is traditionally based on features such as morphologically abnormal lymphoid cells, the presence of lymphoma at other sites, paratrabecular location of lymphoid aggregates within the bone marrow biopsy, and decreased reticulin at the sites of the lymphoid aggregates. Several studies have previously noted these changes [1-5] and it is generally held that the presence of neoplastic lymphoid infiltrates within the marrow in cases of high-grade nodal B cell non Hodgkin's lymphoma conveys a worse prognosis but this is not true for many low-grade B cell lymphomas in which bone marrow infiltration is part of the disease spectrum [1,5-10]. Our aim was to apply quantitative techniques to benign and neoplastic lymphoid infiltrates to establish (i) if objective parameters could be used as a diagnostic tool when assessing bone marrow trephines and (ii) to evaluate differences in nuclear morphometry and morphology between lymph node and bone marrow samples in B cell non Hodgkin's lymphoma.

\section{Methods}

We reviewed all cases of non Hodgkin's lymphoma, excluding chronic lymphocytic leukaemia (CLL), at King's College Hospital, London between the years 1980-1990. Cases of B cell lymphoma were selected for the study (before any treatment had been commenced) which were shown to have both lymph node and bone marrow involvement at presentation. The lymph node appearances were classified according to the modified Kiel classification [11]. Bone marrow involvement by lymphoma was judged using standard histopathological criteria [5].

Reactive lymphoid aggregates within bone marrow were defined as cases in which incidental lymphoid infiltrates had been identified in bone marrow trephines in conditions other than non Hodgkin's lymphoma (Table 1). There was no evidence of lymphoma in these cases, either at the time of diagnosis, or subsequently (duration of follow up 5-14 years).

All tissues used in the study were selected from routine archive surgical histology files and had been fixed in neutral formal saline and processed into paraffin wax. Bone marrow samples had been decalcified in EDTA for two days prior to embedding. Tissue sections 5 micron thick were cut and stained, as a batch, with haematoxylin and eosin (H\&E) using a standardised protocol.

Nuclear morphological and morphometrical studies were performed under oil immersion at $\times 1250$ final magnification using a Zeiss IIIRS microscope. Images were presented via an Ikegami CCD camera to a Colourmorph Interactive Image Analysis System (Perceptive Instruments Ltd., UK) on an

Table 1

Underlying diagnoses in bone marrow trephines containing lymphoid aggregates but showing no evidence of lymphoma

\begin{tabular}{lc}
\hline Diagnosis & Number of cases \\
\hline Cytopaenia & 10 \\
Myelodysplasia & 5 \\
Myeloproliferative disorder & 5 \\
Virus infection & 4 \\
Thrombocytopenia & 3 \\
Other & 3 \\
\hline
\end{tabular}


Elonex 386S microcomputer (Elonex plc, UK) with attached NEC multisync monitor. Morphometric measurements were performed by interactive contouring of nuclear boundaries. Between 100 and 200 nuclei per section were measured, and the mean nuclear area (MNA), standard deviation of nuclear area (SDNA) and range of nuclear size for each sample calculated. The measurement protocols were standardised for each sample.

An assessment of the morphology of nuclear contours was made for each case, samples being graded as composed predominantly (approximately $80 \%$ or more of cells) of round nuclei, cleaved or crenated ("irregular") nuclei, or mixtures of round and "irregular" ("variable") shaped nuclei. Similarly, an assessment of chromatin pattern was made with samples being graded as composed of cells with finely distributed ("uniform") chromatin, coarsely clumped chromatin or a mixture of nuclei showing uniform and clumped patterns ("variable").

Lymph node measurements were performed on lymphoid cells within $2 \mathrm{~mm}$ of the capsule of the node in order to avoid inclusion of incompletely fixed tissue. Areas of necrosis were avoided and sampling was randomly initiated on areas which were representative of the lymphomatous infiltrate. Sequential fields were measured using a battlement technique. Bone marrow samples were measured by initiating sampling at the perceived edge of a lymphoid aggregate and then using a battlement technique to move to sequential fields.

Morphological assessment of the pattern of lymphoid infiltrates in bone marrow was performed at $\times 50$ magnification. The relationship of the infiltrates to bony trabeculae was assessed and the percentage of marrow space occupied by the lymphoid infiltrate was estimated.

Controls: In order to assess the effects of EDTA on nuclear size, fresh lymph nodes from ten cases of B cell non Hodgkin's lymphoma were taken and each node was divided into two halves and fixed in neutral formal saline; one being processed into paraffin wax in the usual way; the other being processed through EDTA according to the schedule for the bone marrow trephines. Differences in mean nuclear area between these "paired tissues" were then measured to estimate the changes produced by EDTA.

To determine the accuracy of subjectively assessing the percentage of marrow space occupied by lymphoid cells, five bone marrow trephines containing lymphoid aggregates were reviewed by the observer who was asked to give an estimate of the percentage of marrow space occupied by the lymphoid cells, to the nearest 5\%. The same trephines were later measured using interactive image analysis techniques and the results were compared with the estimate given by the observer.

\section{Results}

Tissue for histology was available in 535 cases of non Hodgkin's lymphoma (both nodal and extranodal but excluding CLL) at King's College Hospital between the years of 1980-1990. Of these cases 384 were of B cell lineage, 73 were $\mathrm{T}$ cell and 78 were unclassified (immunohistochemistry inconclusive or unavailable). Of the B cell lymphomas, 209 were low grade and 175 were high grade; 119 (58\%) of the low grade lymphomas were considered to be bone marrow positive (with or without evidence of lymphoma elsewhere) on the basis of traditional morphological criteria, whereas only 56 $(32 \%)$ of the high grade lymphomas were bone marrow positive.

82 cases of B cell lymphoma, 44 low grade and 38 high grade, were found to have both lymph node and bone marrow involvement at presentation. The histological subtypes of these cases are shown in Table 2. 
Controls: the mean nuclear areas of lymph node samples processed with or without EDTA are shown in Table 3. These results show that tissues processed through EDTA exhibit some artefactual nuclear shrinkage and may, in some cases, be only $80 \%$ of the size of the corresponding nuclei in tissues not exposed to EDTA.

No differences were found between interactive measurements and observer estimates of the percentage of marrow space occupied by lymphoid aggregates.

Table 4 shows the nuclear morphometric results for reactive and neoplastic lymphoid infiltrates in bone marrow. MNA values for reactive infiltrates and lymphomatous infiltrates in bone marrow show

Table 2

Histological subtypes of B cell non Hodgkin's lymphoma (excluding CLL) at King's College Hospital, London 1980-1990

\begin{tabular}{lcc}
\hline Subtype & Total number & Number BM positive \\
\hline ML small lymphocytic and & 42 & 14 \\
$\quad$ lymphoplasmacytoid/cytic & 76 & 25 \\
ML follicular & 11 & 5 \\
ML mantle cell & 124 & 31 \\
ML centroblastic & 17 & 6 \\
ML immunoblastic & 7 & 1 \\
ML lymphoblastic & & \\
\hline
\end{tabular}

Number BM positive: refers to the number of cases showing bone marrow involvement in addition to involvement at other sites.

Table 3

Control study showing the mean nuclear area (MNA) in square microns of cell samples taken from tissues processed with and without EDTA. The "\% Change" expresses nuclear size in EDTA processed tissue as a percentage of the nuclear size in tissue processed without EDTA

\begin{tabular}{cccc}
\hline Case No. & Without EDTA & With EDTA & \% Change \\
\hline 1 & 24 & 22 & 92 \\
2 & 22 & 22 & 100 \\
3 & 22 & 22 & 100 \\
4 & 23 & 21 & 91 \\
5 & 17 & 15 & 88 \\
6 & 27 & 22 & 81 \\
7 & 25 & 23 & 92 \\
8 & 23 & 24 & 104 \\
9 & 23 & 21 & 91 \\
10 & 21 & 20 & 95 \\
\hline
\end{tabular}

Table 4

Aggregated results of nuclear morphometry in bone marrow samples according to diagnostic groupings - mean (MNA), standard deviation (SD) and range of nuclear area measurements in microns

\begin{tabular}{lcccc}
\hline Diagnosis & Number of cases & MNA & SD & Range \\
\hline Reactive* $^{*}$ Low grade NHL $^{* *}$ & 30 & 14.2 & 1.7 & $10-17$ \\
High grade NHL & 44 & 15.0 & 2.1 & $10-21$ \\
\hline
\end{tabular}

MNA: mean nuclear area; *reactive lymphoid infiltrates; **NHL: non Hodgkin's lymphoma. 
minor differences only. Figure 1 demonstrates the degree of discordance in MNA value between lymph nodes and bone marrow on a "case by case" basis. The mean nuclear areas for the bone marrow samples are smaller than in corresponding lymph nodes in most cases, only 16 of the low grade lymphomas and 6 of the high grade lymphomas showing similarity of MNA in both lymph node and bone marrow specimens. We define "similarity of MNA" in these instances as cases where the MNA for bone marrow cells falls within $20 \%$ of the lymph node sample MNA as defined by the control studies.

Morphological assessment of nuclear contour and nuclear chromatin patterns in bone marrow is shown in Table 5 which suggests that these parameters on their own cannot be used reliably to distinguish between reactive infiltrates, low or high grade lymphomas. The morphological patterns of lymphoid infiltration for reactive and neoplastic lymphoid infiltrates are shown in Table 6. There were no differences between the patterns of infiltration seen in low and high grade lymphomas and some overlap was found between reactive and neoplastic lymphoid infiltrates, although the latter were more often and more consistently paratrabecular, and usually occupied a greater proportion of the marrow space.

\section{Comparing mean nuclear area}

(Lymph node vs. bone marrow)

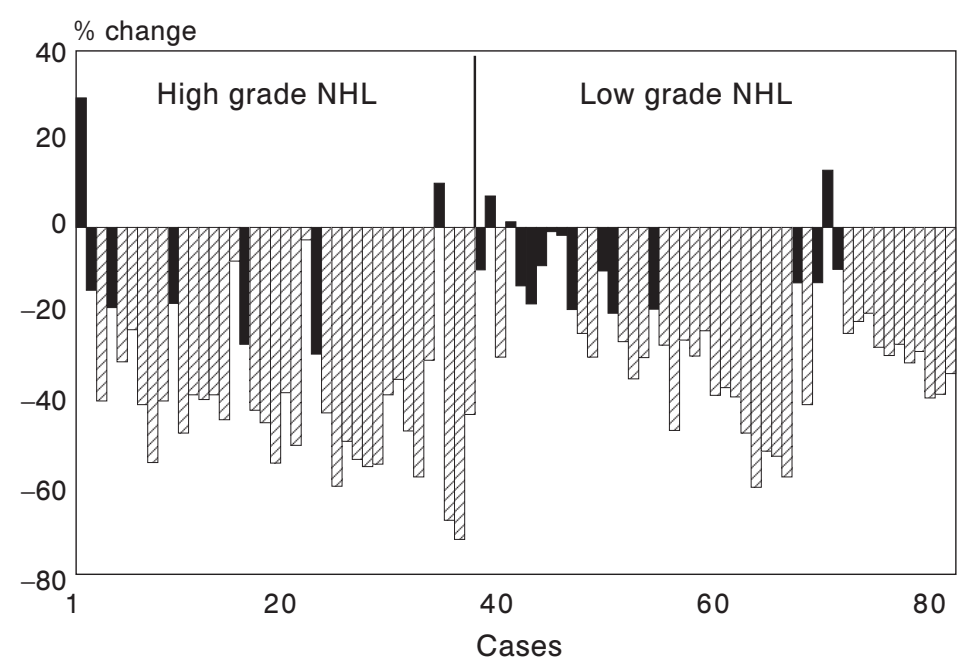

Fig. 1. Differences between mean nuclear area (MNA) measurements from lymph nodes and their corresponding bone marrow trephines in non Hodgkin's lymphoma. The "\% change" denotes the value of the MNA in the bone marrow expressed as a percentage of the MNA in the lymph node. Zero representing identical values in both bone marrow and lymph node; negative values denoting smaller nuclei in bone marrow; and positive values denoting the converse. The cases in black reflect differences which might be explainable on the basis of sampling differences alone (see control studies - Table 3).

Table 5

Morphological appearances of nuclei in bone marrow lymphoid infiltrates. The figures denote the number of cases showing the specified nuclear appearances

\begin{tabular}{lcccccccc}
\hline \multirow{2}{*}{ Diagnosis } & \multicolumn{3}{c}{ Nuclear outline } & & \multicolumn{3}{c}{ Chromatin pattern } \\
\cline { 2 - 4 } & Round & Variable & Irregular & & Uniform & Variable & Clumped \\
\hline Reactive & 5 & 20 & 5 & & 9 & 2 & 19 \\
Low grade NHL & 15 & 26 & 3 & & 12 & 3 & 29 \\
High grade NHL & 7 & 23 & 8 & & 6 & 6 & 26 \\
\hline
\end{tabular}


Table 6

Patterns of lymphoid infiltration in bone marrow ( $n=$ number of cases) and the mean percentage of bone marrow space (\% of BM space) occupied by the lymphoid infiltrates

\begin{tabular}{lccccc}
\hline Diagnosis & Para-trabecular & $\begin{array}{c}\text { Para and } \\
\text { non-paratrabecular }\end{array}$ & Non-paratrabecular & Interstitial/diffuse $\%$ of BM space \\
\hline Reactive & 3 & 3 & 24 & 0 & 5 \\
All lymphomas & 22 & 18 & 16 & 26 & 25 \\
\hline
\end{tabular}

\section{Discussion}

The relatively high percentage of low grade B cell lymphoid infiltrates involving bone marrow, in contrast to high grade infiltrates, is in keeping with the findings of other studies $[1,2,15]$ although the range quoted from study to study is quite wide. MNA values for reactive infiltrates and lymphomatous infiltrates in bone marrow show minor differences which are only discernable using morphometric techniques. These differences are so small that we feel it would be unsafe to use MNA alone as a diagnostic test in many cases, although it may well provide valuable information, lending support to a presumed diagnosis of neoplasia. Similarly, in paraffin embedded tissues, there is considerable overlap of nuclear morphological features between presumed reactive and neoplastic lymphoid infiltrates, making differentiation on these grounds alone unsafe.

As in other studies [8,12-16], we have noted a high degree of discordance between the infiltrates in lymph node and bone marrow (Fig. 1), the lymphoid cells in bone marrow samples often being smaller than the neoplastic cell populations in the lymph nodes. Our control studies suggest that this difference is not due to artefactual shrinkage of cells during processing of bone marrow samples. It seems more likely that different populations of cells are present at the two sites, perhaps reflecting an effect of the microenvironment. The frequency with which papers report discordance $[8,12-16]$ supports this idea. On balance the overall poorer prognosis conveyed by bone marrow involvement in B cell non Hodgkin's lymphoma [1,2,6-10] suggests that the discordant bone marrow lesions are neoplastic infiltrates of low grade, perhaps acting as a reservoir for late relapse following chemotherapy. Some studies have however noted no significant difference in prognosis between cases showing lymph node involvement alone and cases showing lymph node involvement with additional discordant bone marrow infiltrates $[8,17]$. It may be that in some cases, the reported bone marrow infiltrates represent reactive lymphoproliferations accompanying the underlying nodal lymphoma. Immunohistochemistry $[18,19]$, together with the diagnostic features discussed in this study should, however, lend strong support as to whether or not a lymphoid infiltrate in the marrow is truly neoplastic. Significant populations of blast cells were infrequently encountered in bone marrow infiltrates in cases of high grade B cell nodal lymphoma; the lymphoid cells in these cases, being mostly of similar nuclear size, shape and chromatin pattern to the infiltrates seen in low grade lymphomas. From this it might be assumed that it is unwise for pathologists to attempt to predict the histological subtype of any nodal lymphoma on the basis of paraffin embedded trephine material, where lymph node histology is unavailable.

In summary, our results show that in paraffin embedded bone marrow trephines nuclear morphology, nuclear area and the nuclear contour index cannot be used, in most cases, to distinguish reactive from neoplastic lymphoid infiltrates or to classify the subtype of B cell non Hodgkin's lymphoma. In individual cases, however, mean nuclear area can be used to support a diagnosis of a neoplastic infiltrate if it can be shown that the nuclei are larger than would be expected in reactive infiltrates. Such differences are subtle and often not subjectively appreciable. Paratrabecular location of lymphoid aggregates remains one of the best indicators of low-grade lymphoid neoplasia in bone marrow trephines [20]. 


\section{References}

[1] R. Bartl, B. Frisch, R. Burkhardt et al., Assessment of bone marrow histology in malignant lymphomas (non Hodgkin's): correlation with clinical factors for diagnosis, prognosis, classification and staging, Br. J. Haematol. 51 (1982), 511-530.

[2] R. Bartl, B. Frisch, R. Burkhardt, K. Jager, R. Pappenberger and G. Hoffman-Fezer, Lymphoproliferations in the bone marrow: identification and evolution, classification and staging, J. Clin. Pathol. 37 (1984), 233-254.

[3] B.E. Faulkner-Jones, A.J. Howie, B.J. Broughton and I.M. Franklin, Lymphoid aggregates in bone marrow: study of eventual outcome, J. Clin. Pathol. 41 (1988), 768-775.

[4] R. Navone, M. Valpred and A. Pich, Lymphoid nodules and lymphoid hyperplasia in bone marrow biopsies, Acta Haematol. 74 (1985), 19-22.

[5] C. Schmid and P.G. Isaacson, Bone marrow trephine biopsy in lymphoproliferative disease, J. Clin. Pathol. 45 (1992), 745-750.

[6] M. Fraga, P. Brousset, D. Schlaifer et al., Bone marrow involvement in anaplastic large cell lymphoma. Immunohistochemical detection of minimal disease and its prognostic significance, Am. J. Clin. Pathol. 103 (1995), 82-89.

[7] J.G. Gribben, D. Neuberg, M. Barber et al., Detection of residual lymphoma cells by polymerase chain reaction in peripheral blood is significantly less predictive for relapse than detection in bone marrow, Blood 83 (1994), 3800-3807.

[8] G.F. Hodges, T.M. Lenhardt and J.D. Cotelingam, Bone marrow involvement in large cell lymphoma. Prognostic implications of discordant disease, Am. J. Clin. Pathol. 101 (1994), 305-311.

[9] R. Navone, A. Pich, M. Fiammotto and C. Magnani, Bone marrow histopathology and prognosis in malignant lymphoma, Tumori 78 (1992), 176-180.

[10] J.T. Sandlund, R. Ribeiro, J.S. Lin et al., Factors contributing to the prognostic significance of bone marrow involvement in childhood non Hodgkin's lymphoma, Med. Pediat. Oncol. 23 (1994), 350-353.

[11] A.G. Stansfeld, J. Diebold and Y. Kapanchi, Updated Kiel Classification for lymphoma, Lancet i (1988), $292-293$.

[12] R.I. Fisher, R.B. Jones and V.T. DeVita, Natural history of malignant lymphomas at staging evaluation, Cancer 47 (1981), 2022-2025.

[13] K. Foucar, R.W. McKenna, G. Frizzera and R.D. Brunning, Bone marrow and blood involvement by lymphoma in relation to the Lukes-Collins classification, Cancer 49 (1982), 888-897.

[14] P.M. Kluin, J.H. van Krieken, R. Kleiverda and H.C. Kluin-Nelemans, Discordant morphologic characteristics of B cell lymphomas in bone marrow and lymph node biopsies, Am. J. Clin. Pathol. 94 (1990), 59-66.

[15] G. Lambertenghi-Dililiers, C. Annaloro, D. Soligo et al., Incidence and histological features of bone marrow involvement in malignant lymphoma, Ann. Haematol. 65 (1992), 61-65.

[16] G.H. Mead, P. Kushlan, M. O’Neil, J.S. Burke and S.A. Rosenberg, Clinical aspects of non Hodgkin's lymphomas presenting with discordant histologic subtypes, Cancer 52 (1983), 1496-1501.

[17] D. Parker, D.L. Alison, D.L. Barnard et al., Prognosis in low grade non Hodgkin's lymphoma: relevance of the number of sites involved, absolute lymphocyte count and serum immunoglobulin level, Hematol. Oncol. 12 (1994), $15-27$.

[18] R.F. Bluth, T.T. Casey and T.L. McCurley, Differentiation of reactive from neoplastic small cell lymphoid aggregates in paraffin-embedded marrow particle preparations using L-26 (CD20) and UCHL-1 (CD45RO) monoclonal antibodies, Am. J. Clin. Pathol. 99 (1993), 150-156.

[19] J.R. Neilson, J.L. Oates, M. Lumley, M.J. Leyland and J. Crocker, Patterns of bcl-2 staining in bone marrow biopsies from patients with follicular lymphoma, J. Pathol. 175 (1995), 154A.

[20] J.R. Salisbury, M.H. Deverell, J.M. Seaton and M.J. Cookson, Three-dimensional reconstruction of non Hodgkin's lymphoma in bone marrow trephines, J. Pathol. (1997) (in press). 


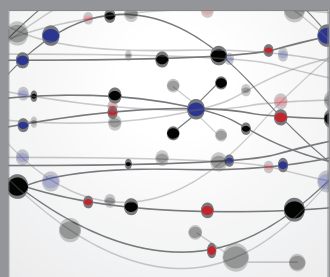

The Scientific World Journal
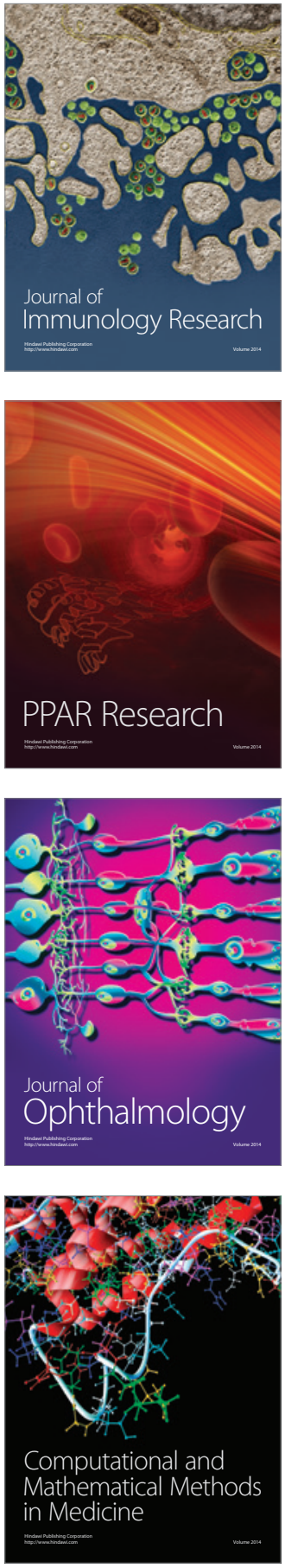

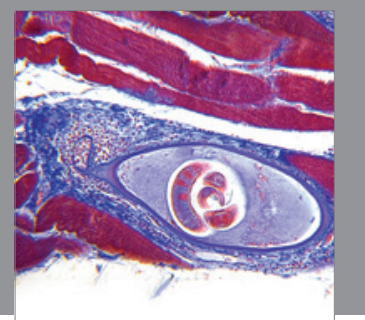

Gastroenterology

Research and Practice
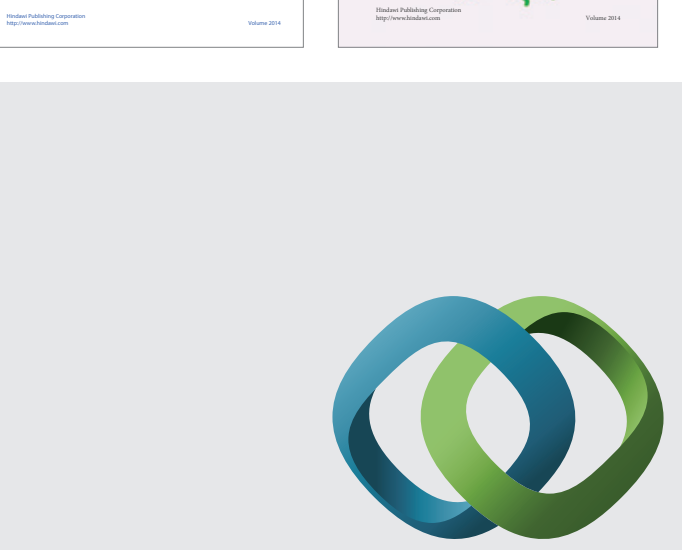

\section{Hindawi}

Submit your manuscripts at

http://www.hindawi.com
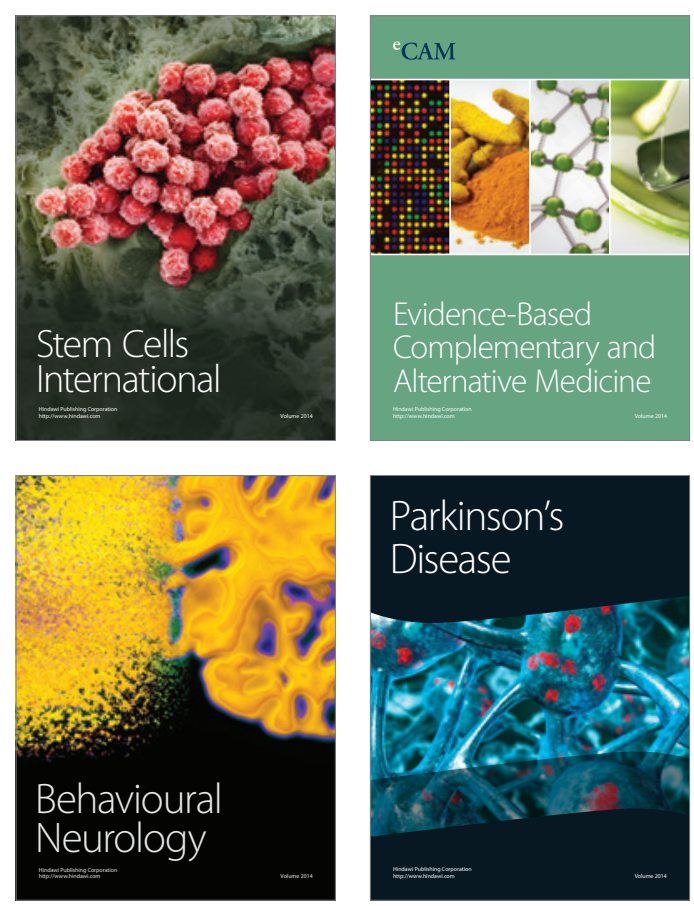

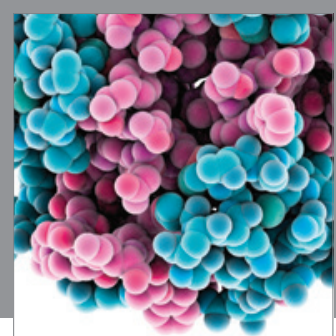

Journal of
Diabetes Research

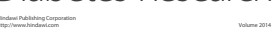

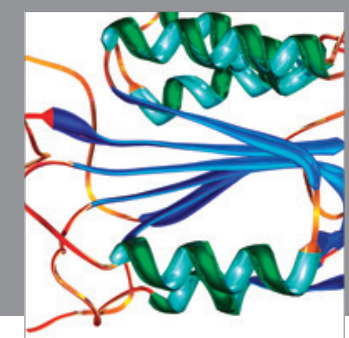

Disease Markers
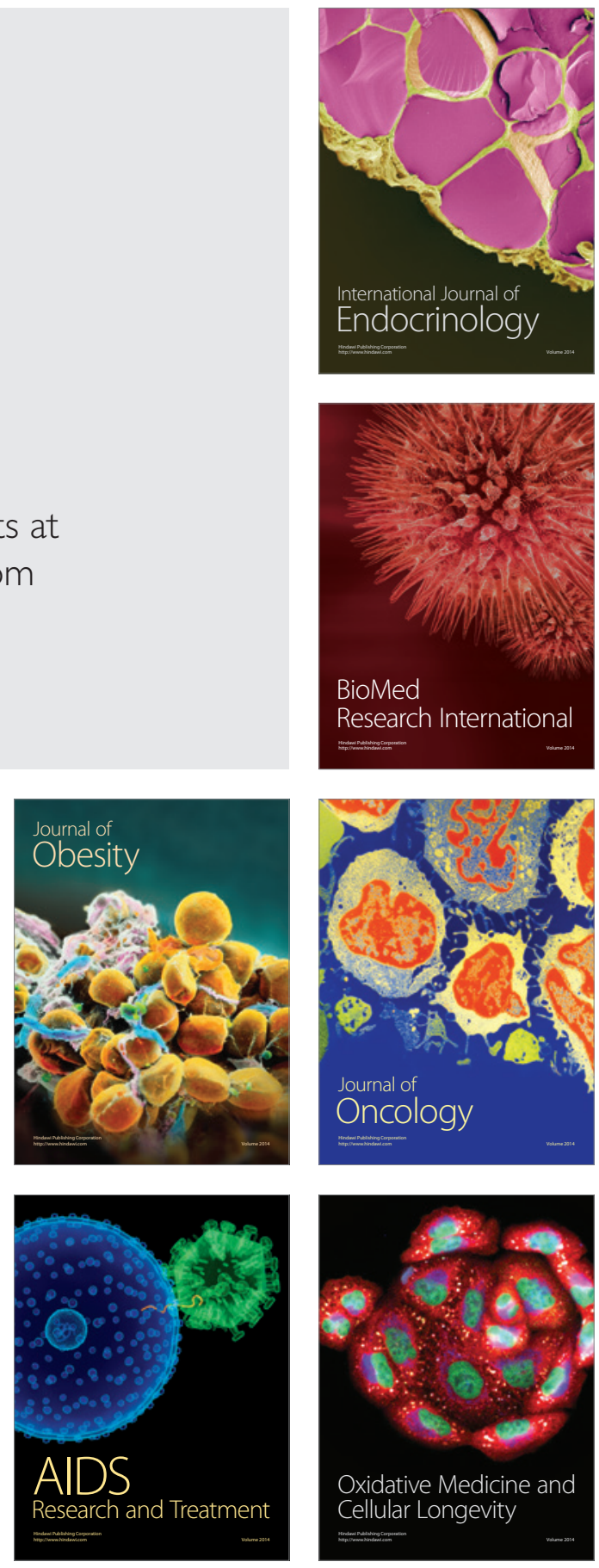